\title{
Stability and electronic properties of ZnSe nanowires: An ab initio approach
}

\author{
Sanjay Prakash Kaushik ${ }^{1}$, Satyendra Singh ${ }^{2} *$, Ram-Krishna Thakur ${ }^{1}$ \\ ${ }^{1}$ School of Basic \& Applied Sciences, G. D. Goenka University, Gurugram - 122103, India \\ ${ }^{2}$ Department of Physics, Seth G. B. Podar College, Nawalgarh - 333042,India \\ *satyendra7171@gmail.com
}

DOI 10.17586/2220-8054-2020-11-5-546-552

\begin{abstract}
The presented work revolves around exploration of the structural dependence of electronic properties of zinc selenide nanowire. For this purpose the shapes under consideration are 2_atom_linear wire, 2_atom_zigzag wire, 4_atom_square wire and 6_atom_hexagonal wire for zinc selenide. ABINIT code has been used for the study. The band structure, geometrical optimization and stability of proposed structures have been studied. A 4_atom_square nanowire structure has come out to be comparatively more stable than other proposed structures while the findings of the study for band structure reveals that zinc selenide nanowires may have conducting, semi conducting or insulating nature which depends on the proposed geometry of the nanowire.
\end{abstract}

Keywords: zinc selenide nanowires, band structure, electronic properties, density function theory.

Received: 10 July 2020

Revised: 25 August 2020

\section{Introduction}

The electrical properties and study of nanowires have been interesting fields to study for the researchers since the 1980's. A drastic variation in optical as well as electrical behavior of these nanostructured substances is seen as compared to their bulk form. The miniature devices show exotic properties and this has increased the need of investigation and exploration of behavior of metallic structure of nanometer-sized dimensions for such devices. These devices seen to have significant advantages over the devices formed by photolithography. The structural dependence of electronic behavior of few nanowires with significant technical importance with implication of local density approximation is the outcome of this proposed work. Extensive experimental work on germanium and silicon nanowires has been already performed by researchers [1-3]. From the family of II-VI group ZnSe semiconductor has its unique application as light emitting diodes [4], photo detector [5] and scintillator [6]. Sufficient experimental work is reported on synthesis of zinc selenide nanowires. Ye et al. [7] synthesized ZnSe nanowire of thickness 20 to 120 nanometer and tens of micrometers in length under an Ar atmosphere by vapor transport. Panda et al. [8] reported that alignment and confinement play a major role in optical properties of synthesized ZnSe nanorods and nanowires. Singh et al. [9] reported the structural dependence for $\mathrm{ZnO}$. ZnSe nanowires with the length of few micronmeters and $80-150$ nanometers in diameter were fabricated and current-voltage characteristics were being studied by Philipos et al. [10]. ZnSe nanowire were being synthesized on the zinc foil by Cheng et al. [11]. Archana et al. [12] used edamine as surface capping ligand and synthesized ZnSe nanowire by wet chemical method. First principles study of optical, magnetic and electronic properties of ZnSe was performed by Benstali et al. [13]. Arya et al. [14] in their work, fabricated ZnSe nanowire via template-assisted electro deposition method. Nasieka et al. [15] studied the doping effect of Er on ZnSe and studied structural properties and found that varying the dopant concentration led to disorder in initial structure. Eitan et al. [16] have performed the guided growth of horizontal zinc selenide nanowires in controlled manner. Zhang et al. [17] studied the first-principles calculations on the basis of DFT investigated the electronic structures and optical properties of $\mathrm{Cr}^{2+}$ zinc selenide. Dong et al. [18] synthesized $\mathrm{ZnSe}$ nanowire via a heat-triggered precursor route. Wisniewski et al. [19] used low temperature spectroscopy to identify the recombination mechanisms for ZnSe nanowire.

The proposed work deals with comparative study of electronic properties, band structure and density of state curve study for four different shapes 2-atom_linear nanowire, 2-atom_zigzag nanowire, 4-atom_square nanowire and 6-atom_hexagonal nanowire, which has not been explored by anyone so far.

\section{Computational details}

The structure of zinc selenide nanowires using DFT calculations [20,21] was explored. This pseduopotential technique is found to be very dynamic tool for studying the electronic and structural properties of different materials [22]. The present study has been performed by ABINIT code [23]. Exchange correlation of Troullier, Martins and Perdew et al. [24,25] has also helped in calculations. The pseduopotential has been obtained from ABINIT Webpage. To test the potentials the calculations were performed on bulk zinc selenide material. The results showed close agreement 
to already existing experimental results. The structures were optimized for Hellmann-Feynman forces up to value of $10^{-3} \mathrm{eV} / \AA \AA$ on each atom. The cut off energy for the calculations has been taken as 30 Hartee. The positioning of nanowire has been done on a super cell along $\mathrm{x}-\mathrm{y}$ direction with side 20 a.u. The $\mathrm{z}$ axis is considered as the axis of the wire. The integration of Brillouin zone has been done and $15 \mathrm{k}$ - points sampling in $\mathrm{z}$ direction has been done with the help of Monkhorst-pack technique [26]. In order to allow atoms to relax no constraints were imposed. The optimized value of lattice parameter for bulk $\mathrm{ZnSe}$ has been obtained. The lowest energy was obtained after due optimization of all the structures.

\section{Results and discussion}

The paper explores the investigation of four completely unmatched geometric structures of ZnSe nanowires. The details of proposed structures have been explored in our already published work [27-35]. The proposed structures of $\mathrm{ZnSe}$ nanowires have been shown in Fig. 1. To get the desired stability the requirement is to get the configuration which belongs to least energy amongst the all proposed structures. The energy variation with inter atomic distance for all geometries is mentioned in Table 1 shows the energy variation with distance for all proposed structures. Table 2 highlights the optimized energy and corresponding distance. The calculation of energy, for infinite linear wire, as a function of $x=a$ has been obtained, wherever $a$ is the zinc selenide distance up to 0.01 nanometers and thereafter the effective total energy is determined. The 2-atom_linear nanowire indicates minimum energy at the $0.64 \mathrm{~nm}$ and acquires stability at $-985.12 \mathrm{eV}$, whereas the 2-atom_zigzag nanowire shows least energy of $-985.29 \mathrm{eV}$ at $0.26 \mathrm{~nm}$ distance. For 4-atom_square nanowire, the energy is least at $0.80 \mathrm{~nm}$ with value of $-985.88 \mathrm{eV}$. The minimum configuration for the 6-atom hexagonally shaped nanowire at the inter atomic distance of $0.38 \mathrm{~nm}$ has lowest total energy, which is found to be $-985.64 \mathrm{eV}$. The overall findings of study show that the 4-atom square nanowire has the least energy configuration as compared other proposed structures under study and this is proposed to be the most stable structure amongst the all. The graph showing the variation between distance and energy, for all the structures, is shown in Fig. 2.

(a)

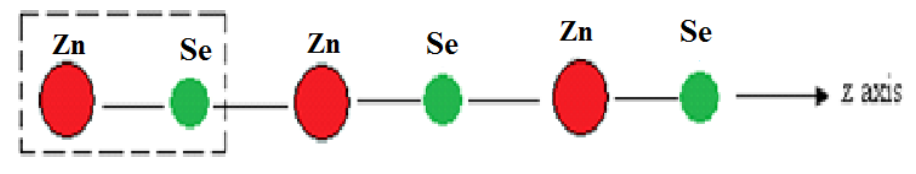

(b)

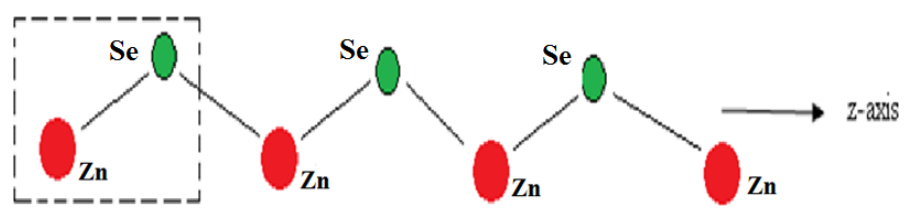

(c)

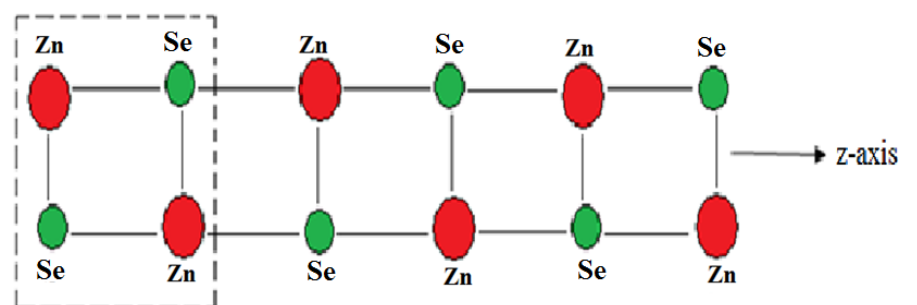

(d)

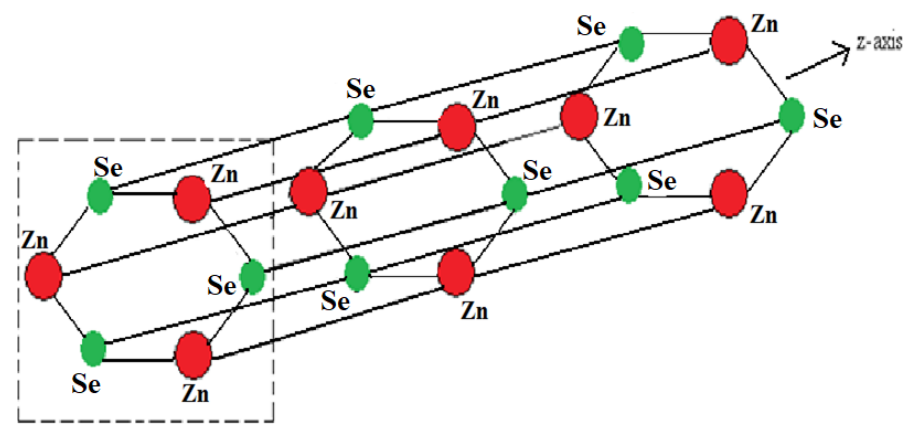

FIG. 1. Structures of ZnSe nanowires: (a) 2-atom ZnSe linear nanowire; (b) 2-atom ZnSe zigzag nanowire; (c) 4-atom $\mathrm{ZnSe}$ square nanowire; (d) 6-atom $\mathrm{ZnSe}$ hexagonal nanowire 
TABLE 1 . The variation between $X$ (interatomic distance in nanometer) vs energy (eV/atom). The bold faced ones are most stable structures

\begin{tabular}{|c|c|c|c|c|}
\hline \multirow{2}{*}{$\begin{array}{c}X=Z n-S e \\
\operatorname{distance}(n m)\end{array}$} & \multicolumn{4}{|c|}{ Total Energy (eV/atom) } \\
\hline & $\begin{array}{c}\text { 2-atom linear } \\
\text { nanowire }\end{array}$ & $\begin{array}{c}\text { 2-atom zigzag } \\
\text { nanowire }\end{array}$ & $\begin{array}{c}\text { 4-atom square } \\
\text { nanowire }\end{array}$ & $\begin{array}{c}\text { 6-atom hexagonal } \\
\text { nanowire }\end{array}$ \\
\hline 0.06 & -558.56 & -707.49 & -288.62 & -277.18 \\
\hline 0.10 & -943.30 & -930.12 & -929.22 & -817.48 \\
\hline 0.16 & -981.71 & -975.71 & -980.68 & -941.08 \\
\hline 0.22 & -983.55 & -983.72 & -985.23 & -972.50 \\
\hline 0.26 & -984.61 & -985.29 & -984.89 & -982.16 \\
\hline 0.32 & -985.07 & -985.00 & -985.31 & -985.14 \\
\hline 0.38 & -979.18 & -983.79 & -984.99 & -985.64 \\
\hline 0.42 & -972.23 & -984.43 & -983.94 & -985.49 \\
\hline 0.48 & -967.21 & -983.78 & -984.34 & -983.57 \\
\hline 0.52 & -977.33 & -985.15 & -984.63 & -974.30 \\
\hline 0.58 & -970.18 & -980.43 & -984.29 & -984.13 \\
\hline 0.64 & -985.12 & -985.12 & -984.91 & -981.84 \\
\hline 0.68 & -979.21 & -977.76 & -985.05 & -878.83 \\
\hline 0.74 & -985.06 & -985.08 & -985.49 & -984.01 \\
\hline 0.80 & -982.18 & -985.16 & -985.88 & -982.87 \\
\hline
\end{tabular}

TABLE 2. The total energy and inter atomic distances of various structures

\begin{tabular}{|c|c|c|c|}
\hline Structures & $\begin{array}{c}\text { Zn - Se distance } \\
\text { (nanometer) }\end{array}$ & $\begin{array}{c}\mathbf{Z n - Z n ~ d i s t a n c e ~} \\
\text { (nanometer) }\end{array}$ & $\begin{array}{c}\text { Total Energy } \\
\text { (eV/atom) }\end{array}$ \\
\hline 2-atom linear wire & 0.64 & 1.28 & -985.12 \\
\hline 2-atom zigzag wire & 0.26 & 0.52 & -985.29 \\
\hline 4-atom square wire & 0.80 & 1.60 & $\mathbf{- 9 8 5 . 8 8}$ \\
\hline 6-atom hexagonal wire & 0.38 & 7.6 & -985.64 \\
\hline
\end{tabular}




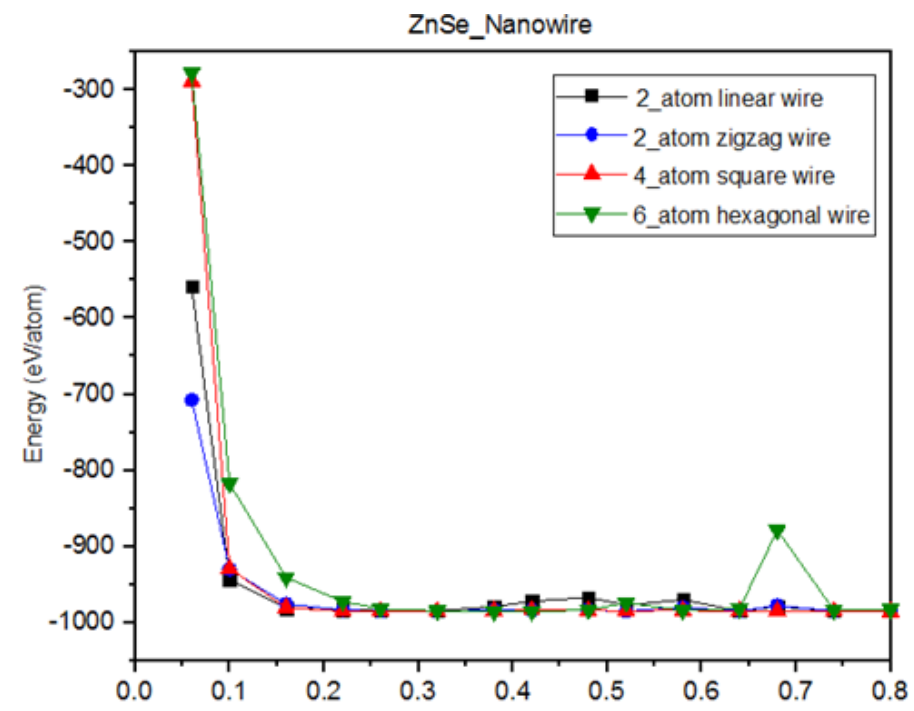

FIG. 2. Variation between interatomic distance $X$ (nanometer) versus energy (eV/atom) for different NWs

The energy versus density of state variation is shown in Fig. 3. The nature DOS for optimized inter atomic distance was under analysis and it shows that the density of state is greater for the 6-atom hexagonal wire near the Fermi level, whereas it is less for the 4-atom squared wire near the Fermi level, and hence, the 4-atom squared shape is the most stable structure amongst those under consideration here.

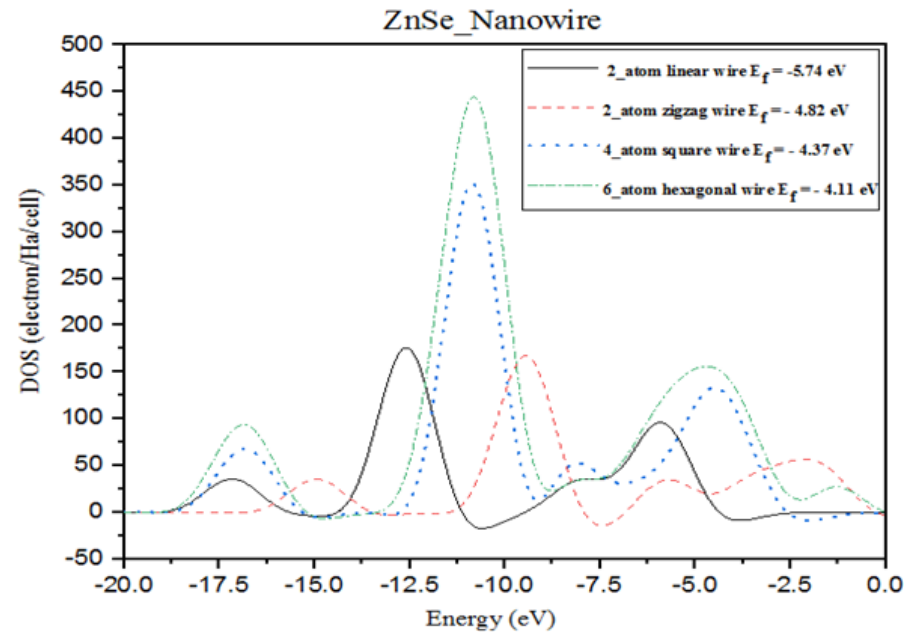

FIG. 3. The variation between DOS versus Energy $(\mathrm{eV})$ with for all structures

The band structures for all the shapes of ZnSe nanowire has been shown in Figs. 4 - 7. Fig. 4 shows that one of the bands crosses the Fermi level for 2-atom linear nanowire, indicating its metallic behavior, whereas for 2-atom zigzag nanowire, one of the bands is lying close to Fermi level as shown by Fig. 5, and thus this structure may be semi conducting in nature. The studies of the band structures for 6-atom hexagonal nanowire and 4-atom square nanowire in Figs. 6 and 7 respectively indicate that all bands are seen to be lesser than the Fermi level, and thus both structures are possibly insulating in nature. 


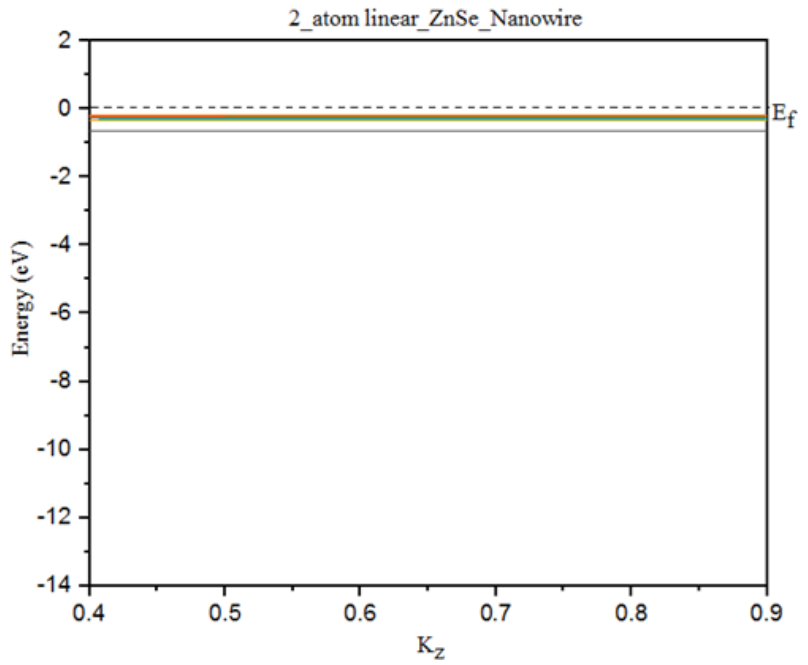

FIG. 4. Band structure for 2-atom linear nanowire

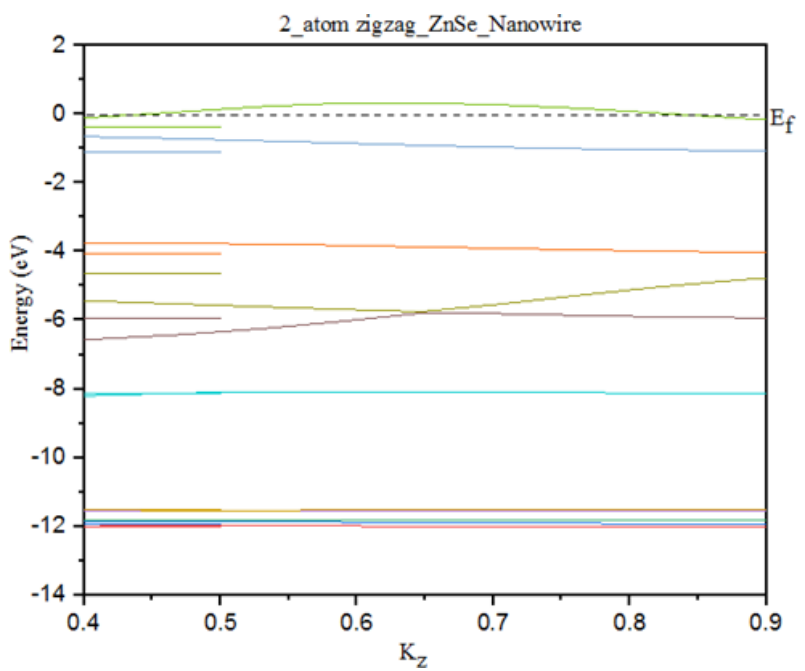

FIG. 5. Band structure for 2-atom zigzag nanowire

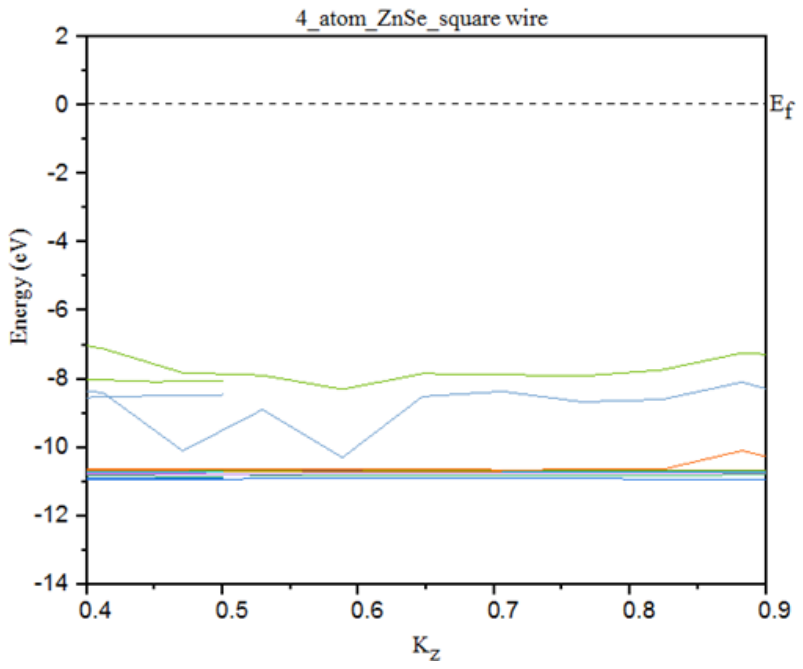

FIG. 6. Band structure for 4-atom square nanowire 


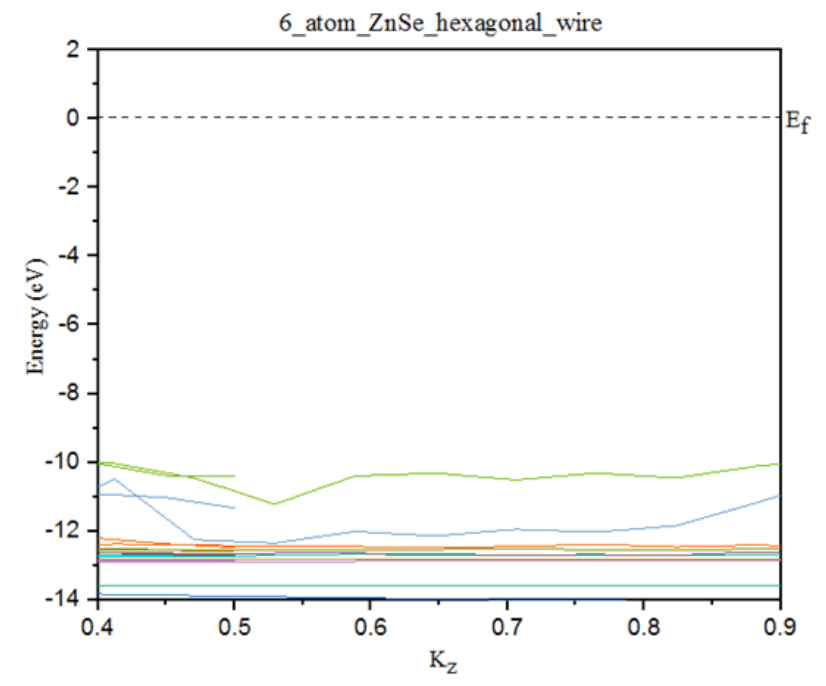

FIG. 7. Band structure for 6-atom hexagonal nanowire

\section{Conclusions}

We have performed an investigation of four different shapes for ZnSe nanowires with ab initio DFT calculations and have analyzed the interatomic distance versus energy, DOS versus energy curve as well the band structure for all the proposed geometries to have better understanding of electronic behavior and stability for the various proposed shapes. The finding establishes that 4-atom square nanowire shape is more stable as compared to other shapes. The bulk $\mathrm{ZnSe}$ is semiconducting in nature, whereas our study for proposed shapes reveals that the 2-atom linear nanowire may be semiconducting in nature. The 2 -atom zigzag nanowire seems to be conducting in nature while the 4-atom square nanowire and the 6-atom hexagonal nanowire is insulating in nature.

\section{Acknowledgements}

We express our gratitude to G. D. Goenka University, Gurugram (Haryana) and Seth G. B. Podar College, Nawalgarh (Rajasthan) for all the help.

\section{References}

[1] LiD., Wu Y., et al. Thermal Conductivity of individual silicon nanowires. Applied Physics Letter, 2003,83 (14), P. $2934-2936$.

[2] Tian B., Zheng X., et al. Coaxial silicon nanowires as solar cells and nanoelectronic power sources. Nature, 2007, 449 (7164), P. 885-889.

[3] Andrew B.G., Lincoln J.L., Mark S.G., Charles M.L. Growth and transport properties of complementary germanium nanowire field-effect transistors. Applied Physics Letter, 2004, 84 (21), P. 4176-4178.

[4] Chen S.H, Wang S.J.J, Lo C.J., Chi J.Y. White-light emission from organics-capped ZnSe quantum dots and application in white-light-emitting diodes. Applied Physics Letter, 2005, 86 (13), 131905.

[5] Vigue F., Tournie E., Faurie J.P. ZnSe-based Schottky barrier photodetectors. Electronic Letters, 2000,36 (4), 352.

[6] Nasieka 1., Boyko M., et al. Optical characterization of Er-doped ZnSe for scintillation applications. Optical Materials, 2014,38 , P. $272-277$.

[7] Ye C., Fang X., et al. Structural characterization of long ZnSe nanowires. Applied Physics A, 2004,79 (1), P. $113-115$.

[8] Panda A.B., Acharya S., Efrima S. Ultranarrow ZnSe Nanorodsand Nanowires: Structure, Spectroscopy, and One-Dimensional Properties. Advanced Material, 2005, 17 (20), P. 2471-2474.

[9] Singh S., Srivastva P. Ab-initio Study of the Structural Stability and Electronic Properties of ZnO Nanowires. Proceedings of fifth international conference "Communication systems and network technologies", 2015, Gwalior, India, April 4-6, 2015, P. 1279-1283.

[10] Philipose U., Ruda H.E., et al. Conducticity and photoconductivity in undoped ZnSe array. Journal of Applied Physics, 2006,99 (6), 066106.

[11] Cheng C.L., Chen Y.F. Low temperature synthesis of ZnSe nanowires by self-catalytic liquid-solid growth. Material Chemistry and Physics, 2009, 115 (1), P. 158-160

[12] Archana J., Navaneethan M., et al. Chemical synthesis of monodispersed ZnSe nanowire and its functional properties. Material Letters, 2012, 81, P. 59-61.

[13] Benstaali W., Bentala S., Abbad A., Belaidi A. Ab-initio study of magnetic, electronic and optical properties of ZnSe doped transition metals. Material Science in Semiconductor Processing, 2013, 16 (2), P. 231-237.

[14] Arya S., Khan S., et al. Electrical properties of electrodeposited zinc selenide (ZnSe) nanowires. Journal of Material Science, 2014, 25 (9), P. 4150-4155.

[15] Nasieka I., Bokyo M., et al. Optical charecterisation of Er doped ZnSe for scintillation application. Optical Materials, 2014,38 , P. $272-277$.

[16] Oksenberg E., Popovitz- Biro R., Rechav K., Joselevich E. Guided growth of Horizontal ZnSe Nanowires and their integration into high performance blue-UV Photodetectors. Advanced Material, 2015, 27 (27), P. 3999-4005. 
[17] Zhang Y., Feng G., et al. Firrst principles study of the electronic structures and optical properties of $\mathrm{Cr}^{2+}$ doped $\mathrm{ZnSe}$ as a function of impurity concentration. Physica Status Solidi (b), 2016, 253 (6), P. 1133-1137.

[18] Li D., Xing G., et al. Ultrathin ZnSe nanowires: one pot synthesis via a heat triggered precursor slow releasing route, controllable Mn doping and application in UV and near visible light detection. Nanoscale, 2017, 9, P. 15044-15055.

[19] Wisniewski D., Byrne K., et al. Fingerprinting Electronic Structure in Nanomaterials: A Methodology Illustrated by ZnSe Nanowires. Nano Letters, 2019, 19 (4), P. 2259-2266.

[20] Hohonberg P., Kohn W. Inhomogeneous Electron Gas. Physical Review B, 1964, 136, P. 864-866.

[21] Kohn W., Sham L.J. Self-Consistent Equations Including Exchange and Correlation Effects. Physical Review A, 1965,140 (4), A1133-A1138.

[22] Martin R.M. Electronic Structure, Cambridge University Press, Cambridge, 2009.

[23] Gonze X., Beuken J.M., et al. First-principles computation of material properties: the ABINIT software project. Computational Material Science, 2002, 25, 478 .

[24] Perdew J.P., Burke K., Ernzerhof M. Generalized Gradient Approximation Made Simple. Physical Review Letter, 1996,77 (18), P. $3865-3868$.

[25] Troullier N., Martins J.L. Efficient pseudopotentials for plane-wave calculations. Physical Review B, 1991,43 (3), P. $1993-2006$.

[26] Monkhorstand H.J., Pack J.D. Special points for Brillouin-zone integrations. Physical Review B, 1976, 13 (12), P. $5188-5192$.

[27] Srivastava P., Singh S., Mishra A. Stability and Electronic Properties of GaN Nanowires - An Ab-Initio Approach. Journal of Computational \& Theoretical Nanoscience, 2008, 5 (4), P. 635-638.

[28] Srivastava P., Singh S. Linear \& Second-order optical response of different GaN Nanowires. Physica E, 2008,40 (8), P. $2742-2746$.

[29] Singh S., Srivastava P., Mishra A. Ab-initio study of Gallium Arsenide Nanowires. Journal of Computational \& Theoretical Nanoscience, 2009, 6 (7), P. 1556-1560.

[30] Singh S.S., Srivastava P., Mishra A. Structural Stability and Electronic Properties of GaSb nanowires. Physica E, 2009, 42 (1), P. 46-50.

[31] Srivastava P., Singh S., Mishra A. Electronic Properties of GaP Nanowires of Different Shapes. Journal of Nanoscience and Nanotechnology, 2011, 11 (12), P. 10464-10469.

[32] Srivastava P., Singh S. Stability Analysis of AlN Nanowire. Journal of Computational \& Theoretical Nanoscience, 2011, 8 (9), P. 1764-1768.

[33] Singh S., Srivastava P. Optical properties of gallium phosphide (GaP) Nanowires. Applied Nanoscience, 2013,3 (2), P. 89-94.

[34] Singh S., Srivastava P. Linear and Nonlinear optical properties of GaAs Nanowires. Applied Nanoscience, 2015, 5 (3), P. $273-279$.

[35] Singh S., Kaushik S.P., Supreet. Ab initio study of Electronic Properties of Cadmium Sulphide Nanowires. J. Comput. Theor. Nanosci., 2020, $17(2 / 3)$, P. 546-551. 